\title{
DETERMINANTES DE LA ORGANIZACION DE LOS ESPACIOS EN LOS CONJUNTOS EDUCACIONALES. UNA EXPERIENCIA CUBANA
}

\author{
(DETERMINING FACTORS IN THE ORGANIZATION OF THE SPACES IN THE TEACHING ESTATES. \\ A CUBAN EXPERIENCE)
}

Ledia Martinez Pillot, Arquitecta

La Habana, Cuba

\section{RESUMEN}

Este trabajo expone criterios sobre determinantes que influyen en el Diseño de Conjuntos, enfocados desde el punto de vista general y conceptual y señala algunos conceptos $e$ instrumentos que permiten medir las relaciones entre los componentes y las etapas que constituyen el proceso de Diseño de Conjuntos Educacionales.

Como parte del trabajo de dichas determinantes, se desarrollan los aspectos siguientes:

- Análisis de criterios para la selección de terrenos: cómo se desarrolla esta actividad en la estructura política y social cubana.

- Tratamiento de los espacios exteriores: como parte de la integración orgánica entre los espacios educativos y demás áreas, para una mayor participación de la vida social y educativa del educando y demás habitantes del centro escolar.

- Análisis de crecimiento: desde la escala urbana hasta los espacios tipos de la Planta Física.

El crecimiento forma parte de los criterios a tomar en cuenta en el proceso de análisis de diseño de conjuntos para que éste pueda mantener su integralidad, considerando ciertos grados de flexibilidad que permita la adaptación a los cambios perspectivos de los sistemas educativos.

Estas determinantes pueden interpretarse y verse reflejadas en los ejemplos que constituyen una experiencia práctica cubana.

\section{SUMMARY}

This work sets forth criteria on determining factors which influence the desing of buildings groups, focused on from the general and conceptual point of view and it points out several concepts and instruments which allow measuring the relations between the components and the stages which constitute the process of designing educational building groups.

As part of the work on these determinants, the following aspects are studied:

- Analysis of criteria for the selection of sites: how this activity is carried out within the Cuban political and social framework.

- Treatment of exterior spaces: as part of the organic interpretation between educational spaces and the remaining areas, for a greater participation in the social and educational life of the pupil and of other inhabitants of the school center.

- Analysis for growth: from the urban scale to physical plant-type spaces.

Growth is a point to be taken into account in the process of analyzing the building group design so that it could maintain its integrity, considering certain degrees of flexibility which would permit adaptation to prospective changes in educational systems.

These determinants can be interpreted and reflected in the examples which constitute a practical Cuban experiment. 


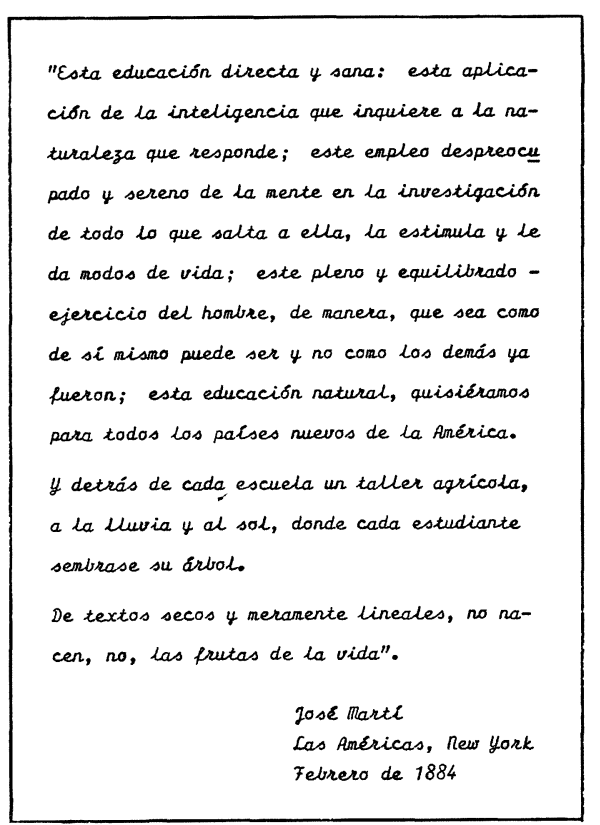

\section{INTRODUCCION}

La arquitectura, mediante su complejo proceso de cristalización, es una imagen de las relaciones sociales que la generan en un momento dado. Por esto, en el proceso dialéctico de transformación que hoy es experiencia concreta en Cuba, se comprueba la validez de un principio revolucionario de la arquitectura.

Sólo la revolución humana es capaz de transformar radicalmente la arquitectura, sólo una vida integralmente diferente demanda la verdadera creación, la arquitectura nueva es respuesta estimulante al desarrollo social revolucionario, esto, unido a las demandas planteadas por un sistema nacional de educación que, a su vez, se encamina hacia la búsqueda de métodos, organización y estructura adecuados a las condiciones propias de nuestra pedagogia revolucionaria, nos lleva a las bases de una nueva arquitectura escolar.

\section{OBJETIVOS}

Los objetivos fundamentales de este trabajo serán exponer criterios sobre determinantes que influyen en el proceso de Diseño de Conjuntos, enfocados solamente desde un punto de vista conceptual y general y que, al mismo tiempo, señalen algunos conceptos $e$ instrumentos que nos permitan medir las relaciones entre los ele- mentos componentes y las diferentes etapas que constituyen el proceso de Diseño, siendo éste un elemento del sistema total del proceso educativo $y$, para su análisis y desarrollo, se debe contar con la participación de un equipo interdisciplinario, en donde las opiniones de los técnicos en materias educativas puedan aportar una valiosa información en el desarrollo del trabajo.

La actividad de Diseño de Conjunto, dentro del sistema Proyectos, tiene relaciones más directas con el sub-sistema de Programación Fisica y el sub-sistema de Análisis de Ubicación. En el primer sub-sistema se obtiene como resultado el Programa Arquitectónico, que implica criterios de organización funcional; en el segundo se obtiene la selección de terrenos y la definición de un emplazamiento.

El sub-sistema de planeamiento y diseño, además de su flexibilidad, debe permitir la individualización de variables que puedan aparecer en el proceso de desarrollo, aún no estando en su inicio. De estas variables, las más definitivas serán las que se convertirán en determinantes en el proceso. Asimismo, de estas variables, las que tienen más influencia en el diseño de conjunto son las siguientes:

- Programa arquitectónico.

- Organización funcional.

- Emplazamiento en el terreno.

Programa Arquitectónico. En esta variable los requerimientos de las actividades educativas se traducen a requerimientos de espacios, asi como el problema educativo se convierte en un problema físico.

Debe ser un proceso permanentemente flexible, pero que permita asimilar los cambios educativos y expresarlos en necesidades cuantitativas y cualitativas de espacios, para que por medio de etapas sucesivas se proceda a analizar el concepto de Crecimiento.

Organización Funcional. La programación arquitectónica de las instalaciones educacionales está constituida por un conjunto de funciones académicas y no académicas, las que aseguran una determinada organización por medio de la cual pueda establecerse un diagrama general de funcionamiento, que será el inicio de la estructura espacial del conjunto.

Emplazamiento. Tiene por objeto la selección de distintas variantes convenientes de localización para cada tipo de espacio necesario para el desarrollo docente de una institución de acuerdo con sus funciones y requerimientos. 


\section{ANALISIS DE COMPONENTES DETERMINANTES DE LA ACTIVIDAD DE DISEÑO DE CONJUNTOS}

\subsection{Análisis de Criterios para la Selección de Terrenos}

La selección de los terrenos para conjuntos escolares corresponde en Cuba al Instituto de Planificación Fisica, pasando por las distintas escalas de planeamiento territorial (Regional, Macro y Microlocalización). La selección se lleva a cabo en base a un estudio socio-económico de cada una de las zonas del pais, el cual tiene como objetivo satisfacer las necesidades de desarrollo integral de cada una de ellas.

En la actualidad, los planificadores regionales en Cuba están centrados en la estructuración de Continuum urbano y en la localización de centros de población, servicios educacionales, etc. sobre la base de que la premisa inherente al desarrollo económico ha de ser la producción agropecuaria, que durante este periodo tendrá supremacia sobre el proceso de industrialización. Esto requiere una transformación en las áreas rurales que, desde el plano social, se proyectan en la dimensión arquitectónica y urbanística, generando los fundamentos de urbanización del campo que no se resuelven solamente por medio de la localización de redes de pueblos (con sus servicios, incluyendo los centros educacionales), sino por la interacción de factores complejos que son un elemento más del conjunto territorial, dentro de las escalas de planeamiento territorial, hasta llegar a los Aspectos de Ubicación, como los que se detallan a continuación:

a) Análisis del Plan Director de la zona con sus componentes, su tiempo de desarrollo $y$ los tipos de inversiones que conformarán dicho plan director. En caso de que no exista ningún estudio de la zona o pueblo, debe realizarse éste antes 0 durante el análisis de ubicación o selección de terreno.

b) Análisis del desarrollo de las zonas, económico y social.

c) Análisis de los terrenos que no están comprometidos en ese desarrollo de la zona, tanto agropecuario como industrial.

ch) Una vez analizadas las distintas zonas, determinamos la necesidad de escuelas y su especialidad en función de los requerimientos del desarrollo económico y social de la zona y de su comportamiento homogéneo en lo concerniente a los aspectos siguientes:

- Densidad de población, escalas y clasificación:
a) en edad escolar;
b) escolarizada;
c) escolarizable.

- Caracteristicas de la zona, rural y/o urbana.

- Tasas de crecimiento.

- Vialidad, comunicación, servicios y además, los estudios de desarrollo de estas tres ramas.

- Hidrografía, topografía.

- Clima: Atención a las condiciones ecológicas de ventilación, iluminación, protección solar y otras, requeridas en Cuba.

Las construcciones se desarrollan en la actualidad en estrecha vinculación y respondiendo a los distintos planes de desarrollo en proceso de realización en el país. Planes industriales, agropecuarios, de educación, de salud pública, de vivienda, de defensa, deportivos y recreativos, de transporte y comunicación, que constituyen los multifacéticos frentes principales de trabajo constructivo a lo largo de todo el territorio nacional. Ya no se trata de resolver el problema constructivo individual, sino de construir para el conjunto de una vida nueva y diferente que abarca la totalidad de la población, razón por la cual la vinculación entre los conjuntos escolares y estos frentes de producción es fundamental para la relación estudio-trabajo.

Por ejemplo, partiendo del análisis de la escala de los asentamientos en concordancia con las áreas de producción y su especialización, se planifican pueblos cañeros de 5/10.000 habitantes, con sus fábricas de azúcar y sus institutos tecnológicos o politécnicos adjuntos; asimismo, pueblos ganaderos de 2.500 habitantes, con el desarrollo de las instalaciones agropecuarias y sus institutos tecnológicos agropecuarios anexos, para los cuales la distribución y escala de los servicios se concibe partiendo no de la estricta rentabilidad, sino de sus funciones como promotores del desarrollo social y de las relaciones humanas y políticas. No cabe duda que la ejemplaridad de esta experiencia cuestionará, en paises en vías de desarrollo en América Latina y otros continentes, un sinnúmero de presiones ahora existentes sobre el proceso de estructuración del territorio. Sólo la opción del cambio radical de las relaciones sociales y de las relaciones de producción configurarán un territorio humanizado concebido como una trama homogénea y coherente.

\subsection{Tratamiento de los Espacios Exteriores, Areas Verdes y Medio Ambiente del Conjunto}

Los espacios y áreas exteriores deben responder a una integración orgánica entre los espacios educativos y demás áreas, para una mayor 
participación en la vida social y educativa de educando y demás habitantes del centro escolar.

Estos espacios exteriores pueden ser clasificados en:

a) Areas deportivas debidamente calculadas y adecuadamente proyectadas en sus condiciones físicas, a la vez que estrechamente vinculadas a los edificios como parte de los espacios circundantes, o formando parte de la comunidad.

b) Areas semi-exteriores, como prolongación de las actividades recreativas y participación de la vida - dormitorio de los educandos y de la comunidad.

c) Areas exteriores de participación social y coeducación, como pueden ser plazas o lugares de estar con un debido estudio de áreas verdes y sus distintas especies, colores del follaje, flores, etc.

Para completar estos aspectos exteriores deben tenerse en cuenta:

- El análisis de los tipos de materiales adecuados para el tratamiento de los pisos exteriores.

- El estudio del color y gráficas en las fachadas de los edificios como complemento del estudio de exteriores en el proceso de diseño de conjunto.

El tratamiento de las áreas exteriores durante el proceso de Diseño de Conjuntos debemos expresarlo dentro de las condiciones ambientales en nuestro pais. Un resumen de la forma que reviste este tratamiento es el siguiente:

- Debe haber coherencia entre los ambientes de la escuela, los ambientes de la vivienda y los espacios de vida.

- El análisis de Conjunto ha de realizarse considerando las condiciones de nuestro pais en cuanto a clima y cultura, así como nuestra condición de pais en vias de desarrollo, liberándonos de los tradicionalismos trasladados mecánicamente del mundo desarrollado y que forman parte del colonialismo cultural y técnico que combatimos, que pueda llevarnos a conclusiones que permitan soluciones ambientales satisfactorias a nuestra sociedad, con un minimo de recursos materiales.

- Ha de plantearse la creación de ambientes planeados para su desarrollo perspectivo según las necesidades de crecer y transformarse con el cambio de las técnicas educacionales, que pueda este ambiente exterior e interior interrelacionarse con el proceso educativo, metodológico, técnico, de producción y desarrollo cultural, vinculándose con la vida de la comunidad.
- Para la materialización de un mejor ambiente escolar se hace necesario el trabajo sistemático de equipos integrados por arquitectos, urbanistas, higienistas, pedagogos, psicólogos, diseñadores gráficos e industriales, escultores y paisajistas.

Según estas premisas, recomendamos que el estudio de estos ambientes exteriores se trabajen en dos escalas simultáneamente: Escala de conjunto y escala de detalles. Además, cuando se elaboran los proyectos de conjuntos escolares prototipos, a cada uno de ellos hay que realizarle el estudio de las condiciones ambientales y de las áreas exteriores.

\subsection{Análisis de crecimiento: Desde la escala urbana hasta los espacios tipos de la Planta Física}

En el Diseño de Conjuntos se ha de considerar cierto grado de flexibilidad que prevea o permita etapas de crecimiento en la planta física y en los espacios tipos, sin que se afecte a la integridad del Diseño, teniendo en cuenta que los procesos académicos son dinámicos e influyen, de manera determinante, en el proceso de análisis de los Conjunto Educacionales.

El crecimiento, entre otras cosas, se puede producir por:

- Cambios de objetivos institucionales.

- Cambios o aumentos de matricula.

- Cambios tecnológicos en la producción que influyan en los centros de educación tecnológica o especializada y que pueden llegar a generar nuevas condiciones de docencia - producción.

El crecimiento forma parte de los criterios a tomar en cuenta en el proceso de análisis del Diseño de Conjuntos, para que éste pueda adoptar una organización tal que responda a zonas como las siguientes:

1. Zona docencia - producción y talleres anexos.

2. Zona administrativa o de dirección.

3. Zona de dormitorios - albergues para alumnos y profesores.

4. Zona de servicios generales.

5. Zona cultural.

6. Zona deportiva.

7. Otras.

Consideramos que el Diseño de Conjuntos debe prever en su estructuración el crecimiento por zonas sin que se afecte su integralidad ni las relaciones funcionales establecidas inicialmente. 
El uso gradual de zonas funcionales complejas y el aumento constante de los requerimientos básicos determinan el desarrollo de sistemas constructivos-susceptibles de crecimiento en todas direcciones y de sistemas de divisiones internas combinadas en diferentes soluciones, todo ello en búsqueda de la flexibilidad necesaria.

El crecimiento puede ser conceptualmente considerado en otras formas, entre ellas la Axil, la Radial y la Celular; esto no cierra la posibilidad de que existan otras modalidades de crecimiento que puedan investigarse posteriormente.

Dentro de los análisis de crecimiento también se estudiará cómo influyen las relaciones entre los espacios en el conjunto y los espacios adjuntos de la comunidad, relaciones funcionales, espaciales, peatonales, vehiculares, visuales, etc.

La universalización de la enseñanza en nuestro país conlleva, en términos urbanisticos, arquitectónicos, constructivos y ambientales, la fusión de los espacios destinados a la educación con las funciones que animan la vida cotidiana de la comunidad en los aspectos culturales y sociales.

\section{ALGUNOS EJEMPLOS DE LA EXPERIENCIA CUBANA}

En Cuba, el Sistema Nacional de Educación está articulado en niveles: primario, medio y superior.

Los sub-sistemas, de acuerdo a su especialidad, han originado programas arquitectónicos y, además, diferentes exigencias para su localización; en este sentido, la vinculación del estudio con el trabajo, como una necesidad para la formación de las nuevas generaciones, se ha convertido en uno de los factores de mayor importancia en la transformación del territorio, pues contribuye a modificar algunas de las caracteristicas sustanciales de la histórica contradicción ciudad-campo.

Los conjuntos arquitectónicos para la enseñanza proyectados y construidos en Cuba en el periodo de 1959 hasta la actualidad, se pueden calificar como grandes instalaciones educacionales que son ejemplos de la aplicación de las Determinantes en la organización de los espacios en el Diseño de Conjuntos Educacionales.

En correspondencia con la estructura educativa del pais, las instalaciones educacionales están agrupadas en construcciones para la:

Educación Primaria, Educación Especial, Educación Media Básica, Educación Media Superior y Educación Superior.
Los ejemplos que a continuación presentamos forman parte de la experiencia cubana, y hemos seleccionado algunos casos representativos de instalaciones educacionales que dan respuesta a la Educación Media Superior y Educación Superior.

\subsection{Primer ejemplo: (Fotos 1 a 8 )}

VOCACIONAL FEDERICO ENGELS, PINAR DEL RIO, CAPACIDAD 2.500 ALUMNOS

Proyectista General: Arq. Ledia Martínez Pillot.

Los objetivos fundamentales en la creación de este tipo de Centro son los de despertar en los jóvenes el interés científico en aquellas ramas de la ciencia y la técnica necesarias al pais y lograr una orientación vocacional y un desarrollo integral en córrespondencia con las demandas actuales del progreso social y la revolución técnica, el aprendizaje laboral y la preparación para la vida acorde con nuestros principios. Estos objetivos fundamentales se conjugan en el programa arquitectónico que sirvió de base para la elaboración de este proyecto.

Partiendo de un proceso de análisis integral de los distintos factores funcionales, ecológicos, topográficos, económicos, técnicos, sociales y culturales que incidian en este tema y en la ubicación especifica de la escuela, surge la sintesis arquitectónica lograda en el proyecto. Este centro educacional se encuentra insertado en la trama urbanistica de la Ciudad de Pinar del Río, con carácter Sub-Urbano, integrado en la futura zona industrial y a la trama agrícola, garantizando asi la combinación estudio trabajo, principio fundamental en el nivel medio de enseñanza.

En su planeamiento de conjunto, el proyecto consta de tres zonas fundamentales que guardan una relación de distancias peatonales entre ellas: la zona escolar, la zona agricola y la zona industrial futura de la escuela. Para lograr esta relación funcional en el terreno asignado fueron necesarios estudios ecológicos, geológicos y topográficos, estudios para la orientación nortesur en los edificios docentes, garantizando asi una iluminación natural adecuada en las aulas, una buena ventilación cruzada en todos los espacios docentes y una orientación este-oeste en los edificios dormitorios, favorables al asoleamiento y a las brisas nocturnas.

La caracteristica accidentada de la topografía fue objeto de análisis, logrando una integración de los edificios a los distintos niveles topográficos, a través de terrazas que garantizan un buen drenaje y uniendo los edificios con escaleras y galerias puenteadas, dando asi posibilidad de circulación entre los diferentes niveles. 


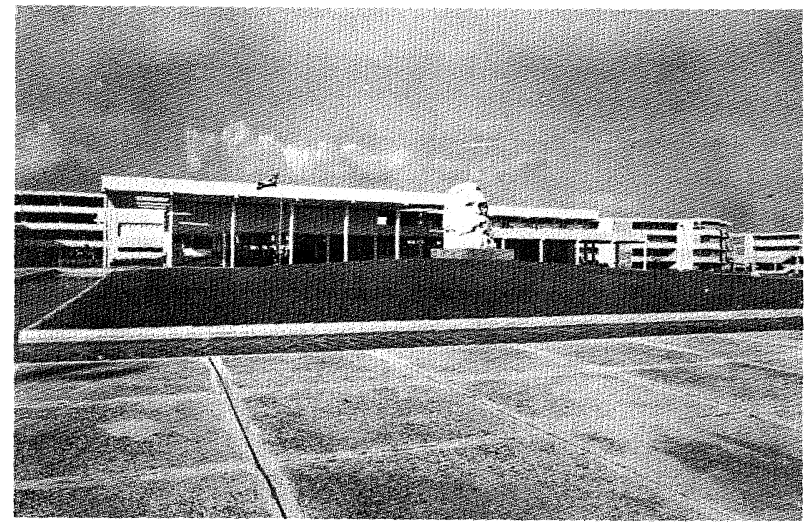
1

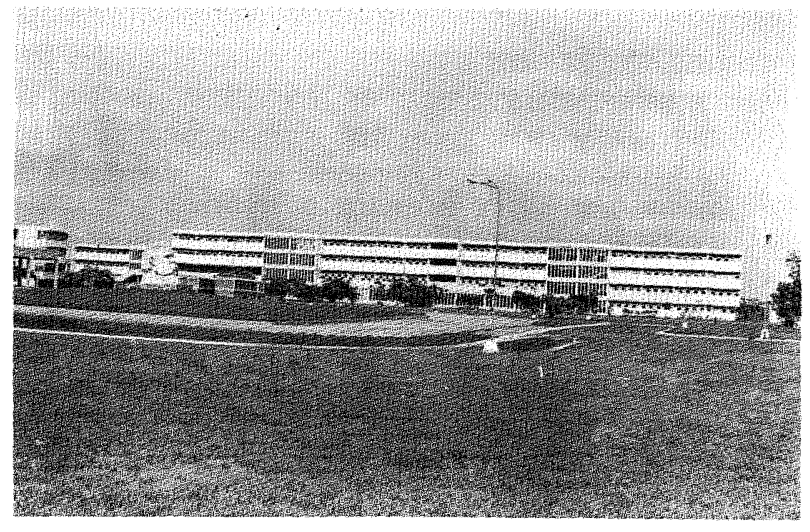
3

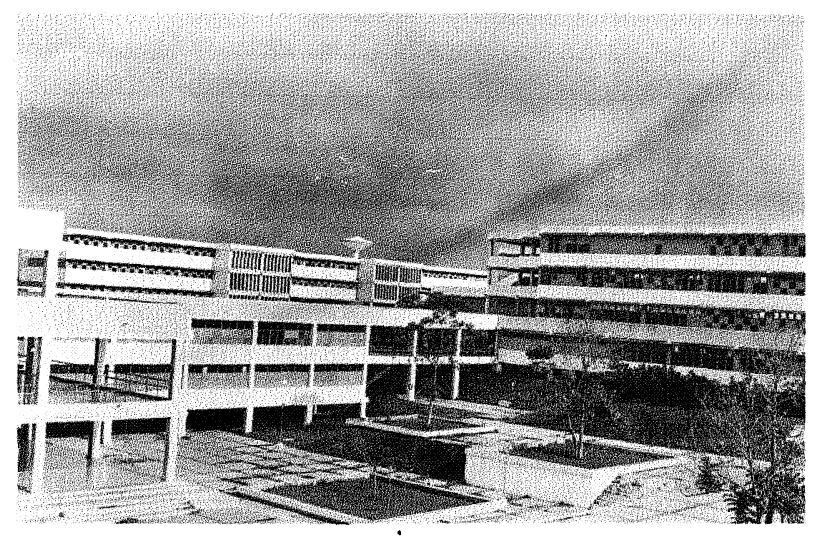

5

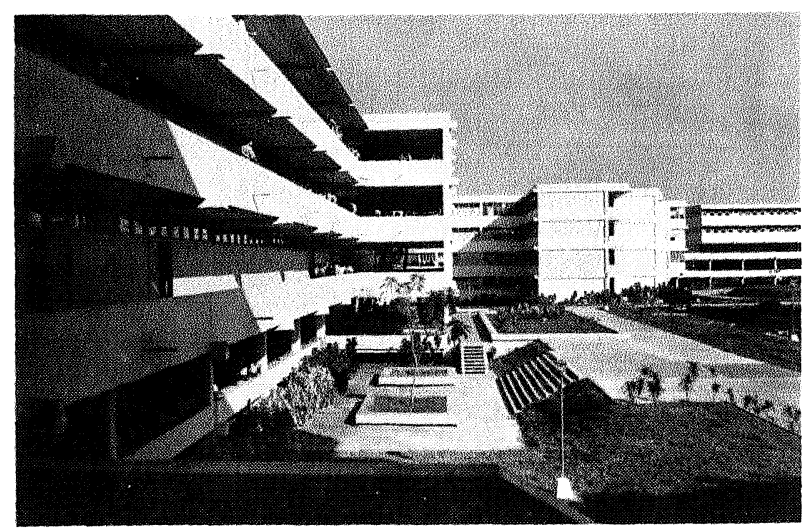

7

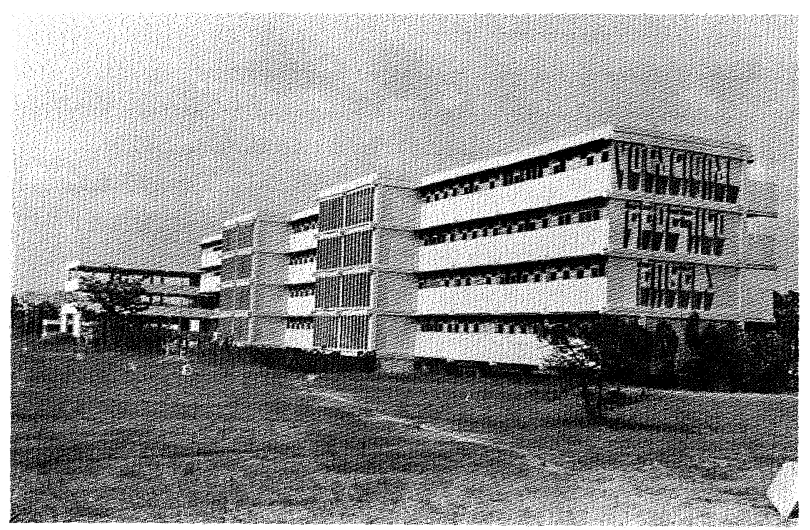

2

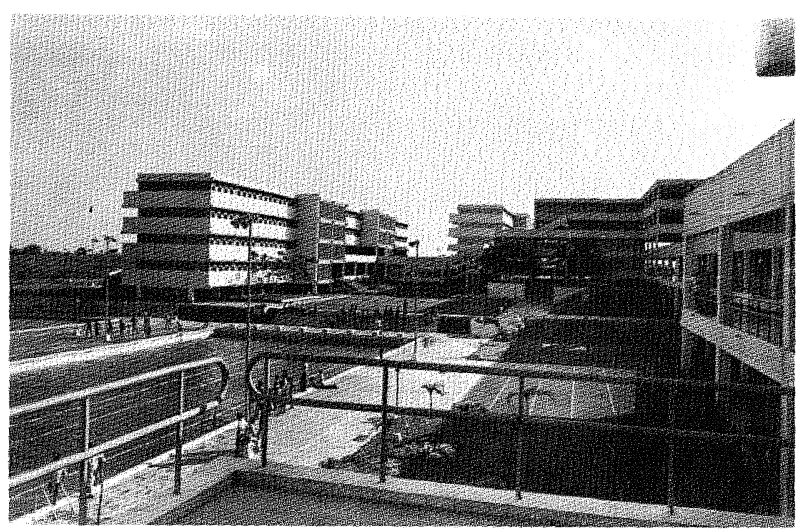

4

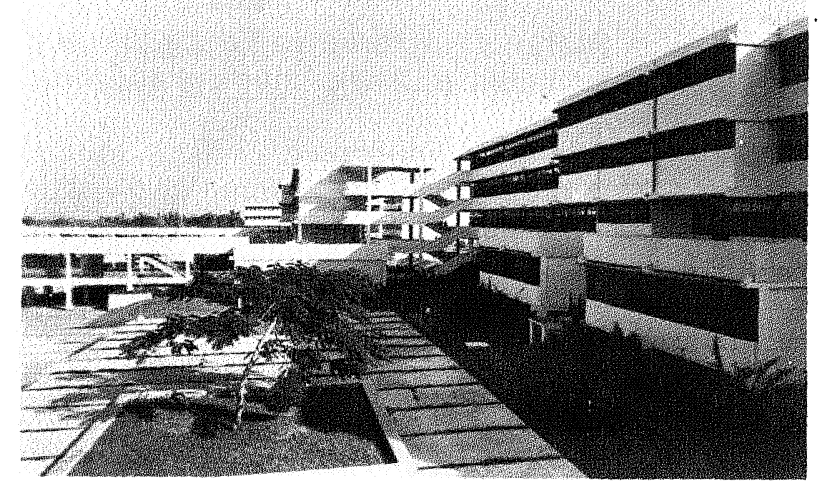

6

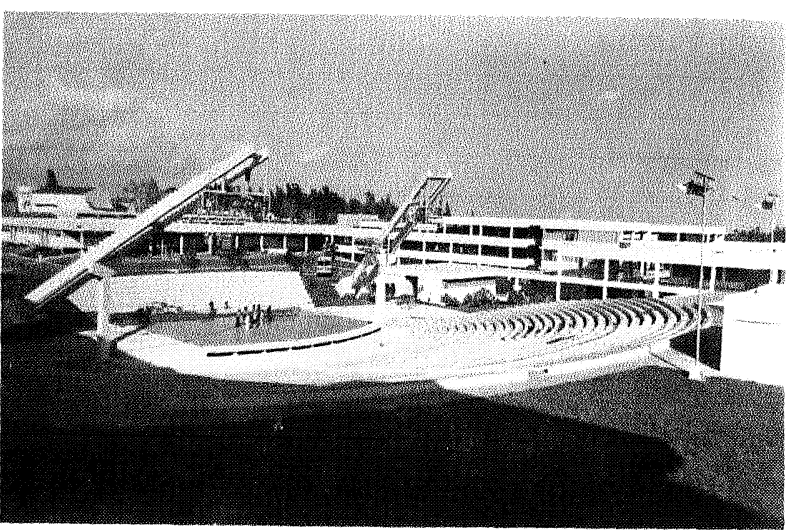

8 
Las relaciones funcionales del Centro determinaron disposición de los edificios de acuerdo a los niveles educacionales a impartir en la escuela, logrando asi un eje virtual central donde se encuentran los edificios con funciones comunes y de servicios generales, al lado derecho las instalaciones propias de secundaría básica y, a la izquierda, las instalaciones propias del preuniversitario.

Otro factor de análisis fue el estudio del color y la gráfica en el conjunto para lograr ambientes y espacios caracterizados de acuerdo a la estructura educacional y a la zonificación de los edificios, con la utilización del color blanco como base de este estudio y el empleo de la policromia en la carpinteria. El tratamiento de color en el conjunto se unifica exteriormente con una banda amarilla que rodea todos los edificios, buscando dentro de las distintas alturas siempre un mismo nivel.

La concepción de relación de diseño entre los espacios arquitectónicos y el diseño gráfico, áreas verdes y de estar, equipamiento, muebles y material de enseñanza forman en conjunto el ambiente de la escuela con la intención de que los factores físicos, biológicos y socio-culturales del medio tengan una influencia decisiva sobre la formación integral del educando.

Del análisis del conjunto de estos factores surgió una sintesis conceptual que alcanza las proporciones arquitectónicas logradas en este proyecto que, unido al objetivo de reducción del área de fabricación, le da el carácter de proyecto concentrado en que se logran espacios entre edificios con diferentes dimensiones, caracteristicas ambientales y usos. Dentro de la sintesis conceptual del proyecto cobra vital importancia la integración entre las artes plásticas y la arquitectura, representada por elementos tales como el busto erigido a la memoria de Federico Engels y que preside el acceso principal a la escuela, ubicado en un domo verde rodeados de un pequeño estanque o linea de agua para lograr una cierta transparencia y frescor en ese ambiente.

Dicha escultura representa la cabeza de Federico Engels con unas proporciones de $3,50 \mathrm{~m}$ de altura y 2,10 $\mathrm{m}$ de ancho, realizada en hormigón fundido in situ con terminación y textura logradas con materiales marmóreos, material propio de la provincia. La organización de los edificios que rodean al busto fue pensada en forma tal que éstas enmarcan el gran espacio principal que preside la escultura como elemento focal esencial de este espacio.

Otro de los elementos plásticos es el Mural en la fachada principal alegórico a la Primera Internacional y el significado de la labor de Federico Engels en el movimiento revolucionario internacional.
El anuncio lumínico con diseño gráfico en las grandes proporciones de sus letras, ubicado en la fachada de uno de los edificios principales, es otro de los elementos plásticos que, unido al busto de nuestro Héroe Nacional José Martí, ubicado en el patio central de este conjunto, dan la dimensión del trabajo conjunto entre el proyectista principal y escultor.

El conjunto de edificios, de acuerdo al programa arquitectónico elaborado por el Ministerio de Educación, está formado por objetos de obra y espacios funcionales tales como:

- Edificio de Dirección General, donde se encuentran ubicadas las oficinas administrativas y de dirección. Biblioteca, Museo y Salón de Exposición.

- Edificio Docente de Secundaria Básica, con las salas teóricas, laboratorios de Biologia, de Física y de Quimica, talleres de Educación Laboral, Privado de Profesores, Almacenes Docentes y otros espacios complementarios como el Centro de Cálculo.

- Edificio de Pre-Universitario, con Aulas Teóricas, Laboratorios de Quimica, de Física y Laboratorios de Biologia, Aulas de Idiomas, Aulas de Clases Facultativas y otros espacios complementarios, tales como Anfiteatro Docente.

- Dormitorios de Secundaria Básica, con áreas de dormir, áreas de estar, áreas de estudio y recreación, locales de recepción de ropa sucia y limpia y otros locales complementarios, tales como un Gimnasio Docente.

- Círculos de Interés y Salones Culturales, con locales de Círculos de Interés, Salón Polivalente, Salón para Exposición, Salón para Guitarra, Salón para Conjunto, Salón para Danza, Salón para Teatro, Salón para Coro, Salón para Artes Plásticas.

- Areas de Servicios Generales, con Cocina Comedores para alumnos y profesores y comedor escuela.

- Almacenes Generales y Talleres de Mantenimiento.

- Casa de Calderas.

- Base de Transporte, para los equipos de la escuela.

- Policlínico de 30 camas, con servicios médicos complementarios.

- Garita de Control, a la entrada de la escuela.

- Tanque de Agua.

- Cisterna.

- Areas Deportivas, con Gimnasio y su anexo para prácticas deportivas bajo techo, terrenos de baloncesto, volley ball, tenis, base ball, balompié, atletismo y un conjunto de piscinas, con una piscina olimpica y otra de entrenamiento y recreación. 
- Areas Complementarias, con Anfiteatro descubierto con capacidad de 3.000 personas y camerinos, Cine Teatro con capacidad de 500 personas.

El proyecto fue elaborado con el Sistema constructivo prefabricado Girón, con elementos y materiales tipificados en su mayoria, buscando una tipologia arquitectónica diferente a través de elementos especificos, tales como la celosia y paneles de barandas de escaleras.

En el proceso de diseño de este proyecto ha primado el concepto fundamental de que esta Escuela reúna las dos ideas que son fundamentales en la formación integral de nuestra juventud: el estudio y el trabajo.

\subsection{Segundo Ejemplo: ESCUELAS FORMADORAS DE MAESTROS}

- Ciudad de Cienfuegos (Fotos 9 y 10)

- Ciudad de Matanzas (Foto 11).

Proyectistas: Fanny Navarrete. Ledia Martinez y Rafael Barbosa, Arquitectos.

Entre las primeras escuelas de 1.000 alumnos que se hicieron se encuentran las de Formadoras de Maestros Primarios de las Ciudades de Cienfuegos y Matanzas.

El objetivo fundamental en la creación de este tipo de centro es desarrollar en los jóvenes el interés en los estudios para Maestros de Escuelas Primarias, combinando el estudio y el trabajo.

Estos objetivos fundamentales se conjugan en el programa arquitectónico que sirvió de base para la elaboración de estos proyectos.

En estos planteamientos de Conjuntos ambas escuelas constan de:

- Zona docente, con la Escuela Primaria Anexa experimental para la práctica docente de los estudiantes.

- Zona de dormitorios de alumnos y profesores.

- Zona de Servicios Generales.

- Zona de Areas Deportivas.

En el caso especifico de Cienfuegos estas áreas deportivas son compartidas con la comunidad. En Cienfuegos, la escuela fue ubicada en el futuro Centro de desarrollo urbano de la Ciudad y se estudio su ubicación de manera que los estudiantes estén a distancias peatonales de las distintas escuelas primarias de la zona en que han de realizar la práctica docente, actividad fundamental en este tipo de Escuelas.

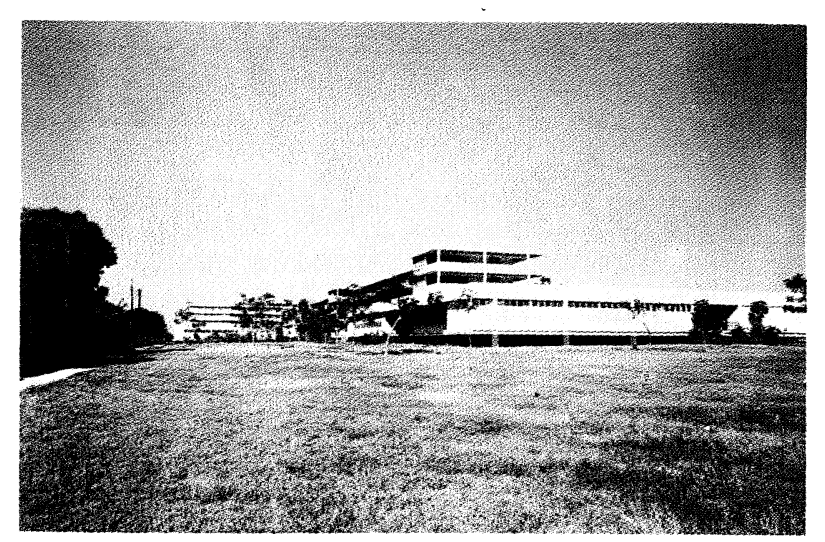

9

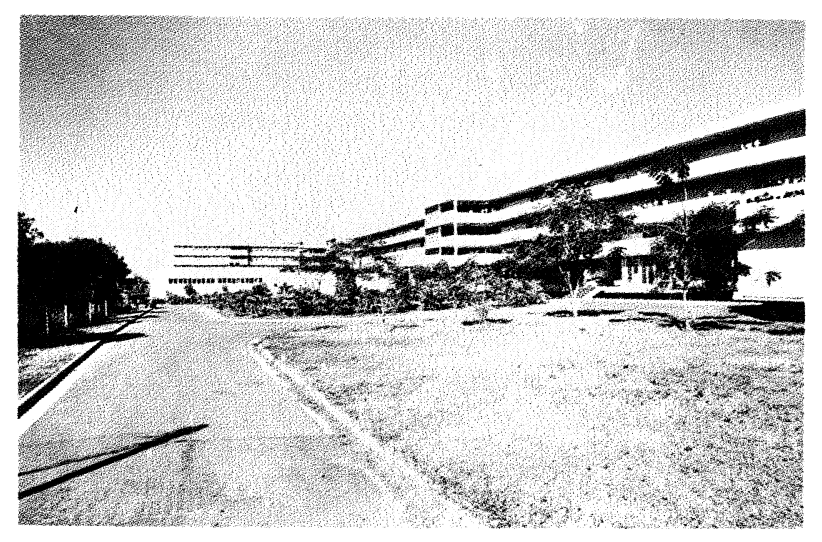

10

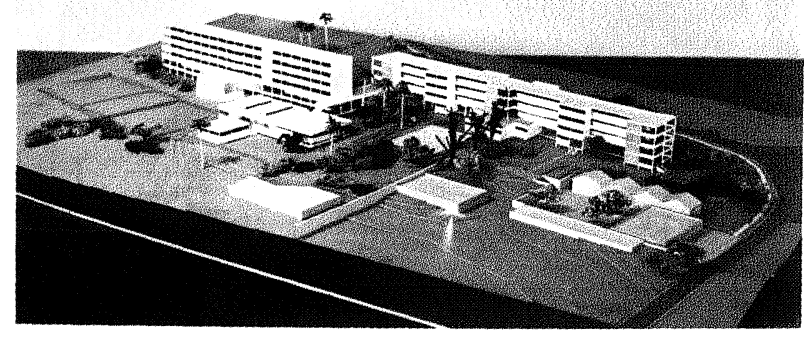

11

En el desarrollo del Diseño están integrados en un conjunto el edificio docente, la escuela primaria anexa y la cocina-comedor y almacén, donde la circulación interior se hace por pasillos y galerias abiertas y semiabiertas.

El desarrollo lineal en cuatro plantas del edificio docente, permite lograr una buena ventilación cruzada en todos los espacios docentes y una orientación favorable hacia el norte, con áreas de sombra en las aulas y en la zona de servicios hasta el sur, favorable al asoleamiento y a las brisas nocturnas. 


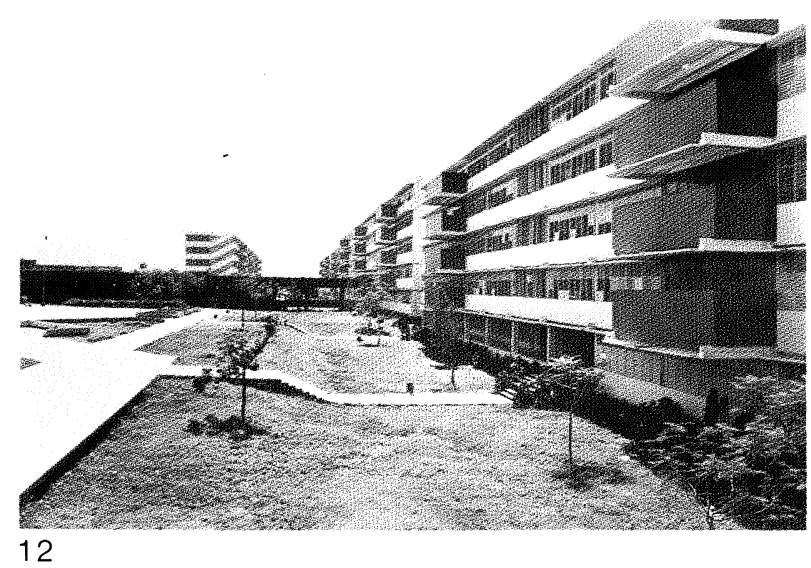

12

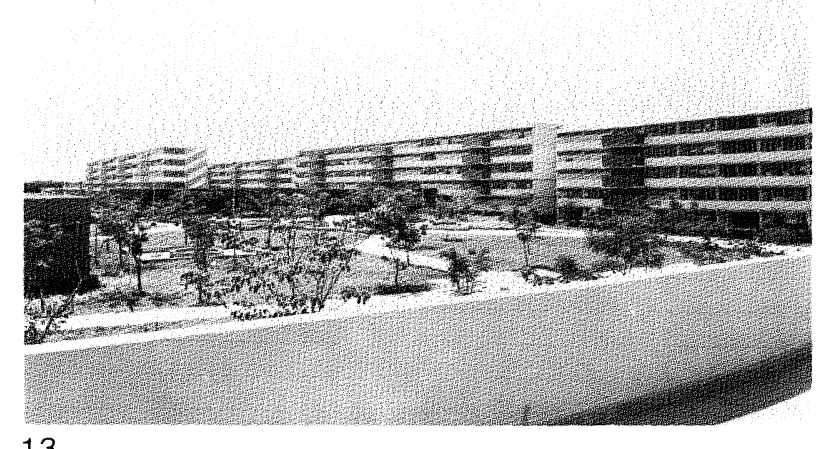

13

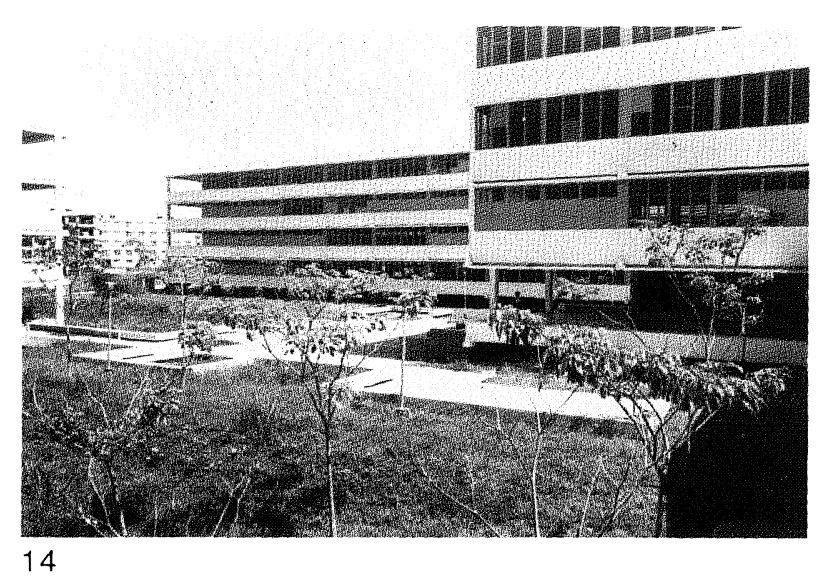

La zona de dormitorios está separada de la zona docente, también a una distancia peatonal, con una orientación este-oeste favorables al asoleamiento y a las brisas. Esta zona se complementa con áreas de servicios sociales, tales como enfermeria, barberia y peluqueria y áreas de servicios complementarios.

En Matanzas la solución es análoga a la de Cienfuegos, resuelta en una topografia más abrupta. El terreno es bien aprovechado por el uso flexible de varios niveles o terrazas, la transparencia de los pisos bajos y el uso intenso de la arborización como protección ambiental.

\subsection{Tercer ejemplo: (Fotos 12 a 14)}

\section{ESCUELA DE FORMACION DE MAESTROS "SALVADOR ALLENDE" para 4.500 ALUMNOS}

Proyectista: Tony Navarrete, Ledia Martinez y Rafael Barbosa, Arquitectos.

Este conjunto Arquitectónico se integra al distrito urbano de Altahabana, en la Ciudad de $\mathrm{La} \mathrm{Ha}-$ bana. Dentro de un área aproximada de 30 ha desenvuelve los bloques docentes y de dormitorios en forma de alas que abrazan un gran espacio continuo, el cual se ramifica en otros más íntimos y conducen a un núcleo de unión de las actividades masivas, conformado por una plaza central y un gran patio cubierto que rodean el cine y el gimnasio deportivo.

Los paseos que intercomunican el gran centro escolar se continúan en el proyecto a través de toda la trama urbana del distrito de la vivienda. Estas alamedas peatonales, acompañadas de arborización en su recorrido, unen a los maestros con sus escuelas primarias y recorrer los centros comunales de los microdistritos y distritos, sin interferencias de tránsito, por áreas protegidas del ruido y de las emanaciones para el pleno disfrute peatonal.

\subsection{La Enseñanza Superior en Cuba}

Como parte del sistema educacional, la universidad se integra a los planes generales de desarrollo vinculándose, según las necesidades de los mismos, a través del planeamiento regional y nacional, a la comunidad en transformación.

La incorporación de las funciones de docencia e investigación de la educación superior a los procesos productivos del pais, se traduce en la integración de los espacios universitarios nacionales de desarrollo.

El objetivo a alcanzar en el proyecto de una instalación educativa de nivel universitario no es solamente alojar, en forma adecuada, a hombres y funciones nuevas en edificaciones cuya solución arquitectónica se aproxime a los estándares ya establecidos y en proceso de desarrollo en el mundo.

La meta real es mucho más amplia: hacer que las universidades contribuyan a resolver las necesidades y aspiraciones progresivas de la nación y ayuden a forjar a los integrantes del más alto grado científico del país. El Diseño de Conjuntos tiene que relacionarse con los enfoques revolucionarios cerca de la vida universitaria y los planes de estudios, que plantean variados y complejos requerimientos a todos los niveles del programa de inversión, proyección, ejecución y funcionamiento ulterior de la obra. 
La flexibilidad es un ingrediente necesario tanto en los conjuntos como en los espacios; una nueva universalidad debe ser planificada para posibles aumentos y transformaciones, ya que la rigidez espacial en ellas ocasiona graves trastornos y grandes desembolsos económicos posteriores.

A esta flexibilidad debe responder dentro del Diseño de Conjuntos, el estudio del Edificio, su estructura, formando parte del subsistema estructural, tratando de que el sistema óptimo sea el que permita, además, una amplia gama de aplicabilidad a distintos programas, asi como adecuación flexible a futuras situaciones educacionales.

Del estudio comparativo de las diferentes soluciones que se han elaborado para distintos conjuntos universitarios, se concluye que éstos se agrupan según dos criterios principales, los que presentan un desarrollo de sus edificios en una gran extensión y aquéllos de composición compacta, con tendencia a plantear relaciones más estrechas con el hombre, sin perder la jerarquia de las edificaciones, de acuerdo con la codificación urbanistica y el análisis de las Determinantes en el Diseño de Conjuntos. Entre los más recientes podemos citar como ejemplos algunos de estos conjuntos universitarios:

- Centro Universitario en las Ciudades de Pinar del Río y Holguín.

- Instituto Superior de Ciencias Agricolas en Bayamo.

- Instituto de Ciencias Médicas en Villa Clara.

- Instituto Sidero - Minero - Metalúrgico en Moa.

- Universidad de Camagüey.

- Instituto Superior de Ciencias Agropecuarias en las Ciudades de La Habana y Ciego de Avila (Fotos 15 a 18).

En este último ejemplo podemos analizar las condiciones propias de nuestros requerimientos, en el que, de acuerdo con las disponibilidades de recursos, se enfocó el Diseño de Conjunto en el campo de las soluciones compactas adecuadas a los programas especificos y se ha mantenido como motivación principal crear en el seno del conjunto las instalaciones arquitectónicas indispensables para el desenvolvimiento inherente a la vida de la población estudiantil.

Partiendo de las necesidades a satisfacer del estudiante: estudio, trabajo, alimentación, recreación y cultura y descanso, se trazó un esquema de la organización de las edificaciones alrededor de un punto focal, la plaza, que constituye el centro del conjunto urbano. Teniendo en cuenta las características climáticas y físico-

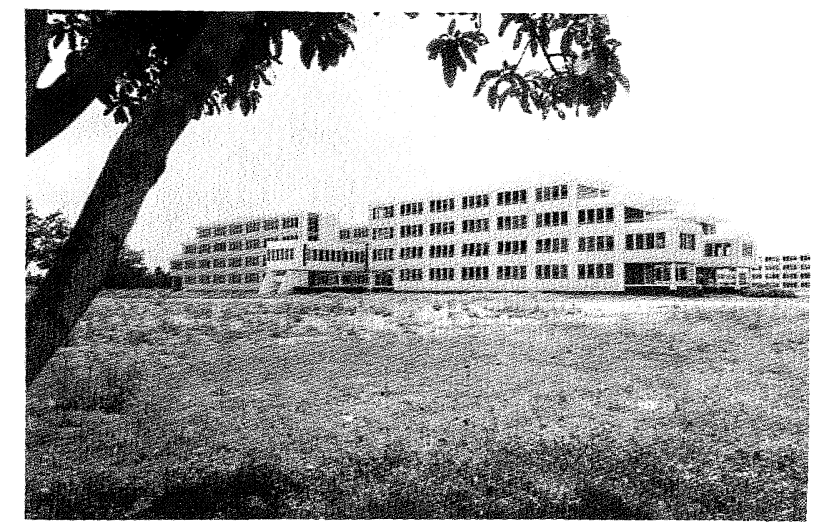

15

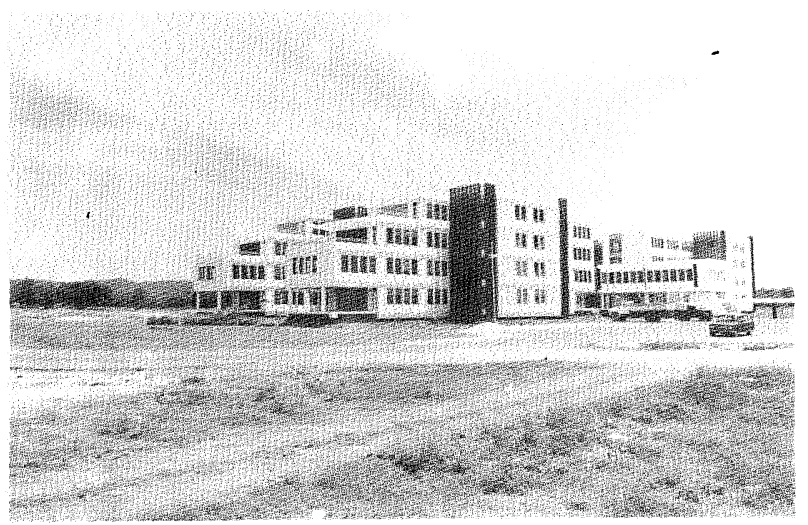

16

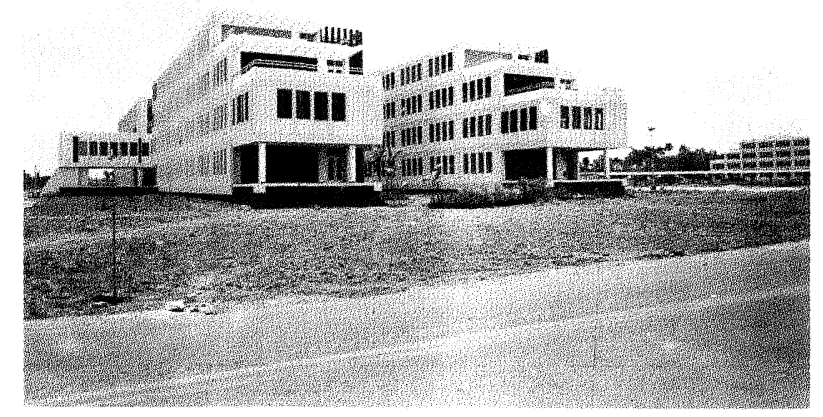

17

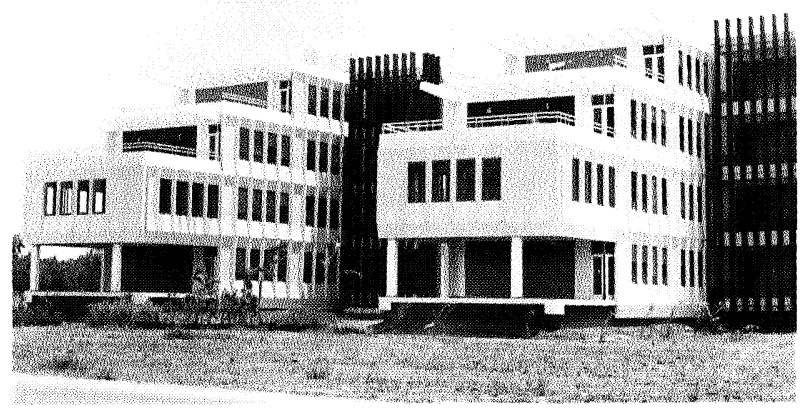

18 
ambientales de la zona de microemplazamiento, se ubicaron las edificaciones de forma propia para el asoleamiento y las brisas nocturnas.

Este Diseño de Conjunto está conformado por edificios de albergues, centro comercial, para actividades sociales y de servicios generales: aqui se dispone de comedores, cafeterías, biblioteca, los espacios sociales de la juventud, talleres de aficionados, tiendas, locales de servicios, etc.

En el lado occidental del conjunto se ha creado el área docente, que incluye anfiteatro, museos, laboratorios, talleres y edificos para profesores, espacios de investigaciones, etc. Todo este conjunto se desarrolla en dos niveles, con comunicación rápida por medio de una galeria-puente que sirve de interrelación entre diferentes zonas del conjunto.

Tal tratamiento de los niveles y de la disposición relativa de los edificios y áreas libres facilita, además, la creación de distintos espacios y ambientes diferentes, lo cual permite ofrecer gran animación y evita la monotonia.
Completan el conjunto las áreas deportivas y las extensas áreas de polígonos agricolas para la práctica docente.

Las ideas revolucionarias basadas en un método científico en un ambiente estimulante para la creación, son las caracteristicas a que aspiramos en nuestra futura escuela y en nuestra comunidad.

«La Educación es lo más importante que este pais tiene que hacer, después de haber hecho la Revolución".

\section{BIBLIOGRAFIA}

- Conferencias - Determinantes de la Organización de los Espacios en los Conjuntos Arquitectónicos.

Expositor: Arq. Eugenio G. Cáceres.

Conescal, Méjico - 1975.

- La Arquitectura Escolar de la Revolución Cubana. Ponencia presentada por Cuba en el Seminario Internacional de Construcciones Escolares DESA, en La Habana - octubre, 1973.

- Revista Arquitectura, Cuba. N.o 347-48, año 1978. 